\title{
Effects of substorms on the stormtime ring current index Dst
}

\author{
Gordon Rostoker \\ Solar-Terrestrial Environment Laboratory, Nagoya University, Toyokawa, Japan \\ e-mail: rostoker@stnet1.stelab.nagoya-u.ac.jp
}

Received: 25 February 2000 / Revised: 31 May 2000 / Accepted: 4 July 2000

\begin{abstract}
There has been some discussion in recent times regarding whether or not substorm expansive phase activity plays any role of importance in the formation of the stormtime ring current. I explore this question using the $K p$ index as a proxy for substorm expansive phase activity and the Dst index as a proxy for symmetric ring current strength. I find that increases in Dst are mildly related to the strength of substorm expansive phase activity during the development of the storm main phase. More surprisingly, I find that the strength of Dst during the storm recovery phase is positively correlated with the strength of substorm expansive phase activity. This result has an important bearing on the question of how much the Dst index reflects activity other than that of the stormtime symmetric ring current strength for which it is supposed to be a proxy.
\end{abstract}

Key words: Ionosphere (electric fields and currents) Magnetospheric physics (current systems; storms and substorms)

\section{Introduction}

It has been known for some time that the negative $\mathrm{H}$ component depressions of up to several hundred nT observed at low-latitude magnetic observatories are due to large-scale currents flowing around the Earth carried by gradient/curvature drifting charged particles (Singer, 1957). These negative H-component storm disturbances can last for several days and comprise a main phase of up to several hours during which the disturbance strengthens and a recovery phase of up to many days during which the disturbance returns to its pre-storm level. Storms are often preceded by a sudden increase in the $\mathrm{H}$-component called a sudden storm commencement (ssc) followed by a period of relative quiescence called the initial phase which may last some hours before the main phase begins. Neither of these two disturbances is an essential ingredient for a storm. Whether or not a storm is considered to have taken place depends solely on the strength and, to some extent longevity, of the negative H-component disturbance as detected globally. Sugiura (1964) designed an index called Dst which provides a measure of the global character of the negative H-component perturbations and which is presented as a proxy for the symmetric component of the ring current. It should be emphasized that the term "symmetric" is more mathematical than physical in its meaning. The storm time ring current is much more complex in character than the term "symmetric" might imply (see Lui et al., 1987). Quantitatively the Dst index is calculated to be approximately the average value of the H-component disturbance seen at (nowadays) four low-latitude stations well distributed in longitude. There is no minimum threshold above which one has a physical basis to claim that a storm is in progress: however, for operational purposes in space weather forecasting, a storm is considered to have occurred when Dst exceeds $\sim 50 \mathrm{nT}$ (Joselyn and Tsurutani, 1990).

In recent years, a controversy has arisen as to the nature of the physical mechanism directly responsible for the origin of the symmetric ring current. Whatever that mechanism may be, it must be capable of accelerating protons to energies of $\sim 100-200 \mathrm{keV}$ as these energetic ions appear to be the primary ring current carriers for the symmetric ring current. Until fairly recently, it was thought that substorm expansive phase (EP) processes must be responsible for ring current particle acceleration since substorm activity seems to be the unique feature associated with ring current growth during the storm main phase (see Akasofu and Chapman, 1961). However, in the 1970's a school of thought developed which suggested that the only important factor for ring current development was the convection electric field imposed from the interplanetary medium through the magnetic merging process (Burton et al., 1975).

The question has come into focus more recently through the contention of Iyemori and Rao (1996) that 
substorm expansive phases are not responsible for symmetric ring current growth and, in fact may limit that growth. Indeed, Rostoker et al. (1997) have found evidence that some large substorm expansive phases, even occurring at low latitudes, seem to have little impact on the growth of the symmetric ring current index Dst. There is therefore good reason to study the response of $D s t$ to substorm EP activity and my purpose here is to explore that question further.

\section{Processing of the data and presentation format}

In this study I use the Dst index as a proxy for symmetric ring current strength and $K p$ as a proxy for substorm EP activity. The stations that contribute to the $K p$ index are primarily located at middle latitudes. Under normal circumstances, the value of $K p$ is a measure of the field-aligned currents flowing into and out of the auroral oval with the largest values generally occurring during substorm EP activity. Under storm conditions, the auroral electrojets may expand far equatorward and some contribution to $K p$ may come from ionospheric rather than field-aligned currents. This would, for our purposes introduce a source of error into our analysis as it would sometimes lead to larger values of $K p$ than would be registered if only field-aligned current were contributing to the index.

I should note at this time that some workers have chosen to use $A E$ or $A L$ as a proxy for substorm activity (e.g. Cade et al., 1995). It would appear, on the surface, that using a one hour index ( $A L$ or $A E$ ) to correlate with a one hour index (Dst) would be a more favourable approach than using the three hour $K p$ index. However, I would argue that $A E$ or $A L$ are unsuitable for investigating the storm-substorm relationship because it is really the role of the EP which is at issue when it comes to ring current formation. Although the driving electric field may be important in the transport of energetic particles during a storm main phase as suggested by Burton et al. (1975), the electric currents that flow as its consequence only serve to dissipate energy in the ionosphere that might have otherwise been available for ring current formation. A significant portion of the $A L$ or $A E$ disturbance can therefore be attributed to directly driven activity which cannot really be associated with ring current growth. In fact, these indices actually describe energy that is being dissipated in the auroral oval ionosphere and which is, therefore, unavailable for ring current formation. In contrast, $K p$ is primarily caused by the field-aligned currents associated with the substorm current wedge. Accordingly, it is the best available index for establishing the level of substorm EP activity during the growth and decay of magnetic storms even if it is a three hour index.

It is useful to note here that Campbell (1979) has investigated the relationships among the parameters $D s t, A E$ and $a p$ (where $a p$ is the linear counterpart of $K p$ ). He found a linear correlation coefficient for $A E$ and $a p$ of 0.89 , which in recent time would be attributed to the fact that high levels of directly driven activity are normally (but not always) accompanied by strong substorm expansive phases.

Because $K p$ is a three hour index and $D s t$ comes as hourly values, it was necessary to average the three values of $D s t$ in an interval covered by one value of $K p$. Our study involves establishing the relationship between the values of $D s t$ and $K p$ in a given three hour interval as well as the relationship between the amount of change in $D s t[\delta(D s t)]$ in each three hour interval corresponding to a given $K p$. The latter relationship was explored on the assumption that the larger the level of substorm activity might be, the larger would be the increase in symmetric ring current strength.

I looked at all magnetic storms over the interval 1987-1996 inclusive, breaking down the data into periods of main phase growth of the stormtime ring current and periods of decay of that ring current. As will be seen later, I also found it necessary to break down the recovery phase data into periods early in the recovery phase and periods later in the recovery phase. I established a set of selection criteria so as to minimize the scatter in the data that one would expect from disturbances which can develop in such a highly variable fashion. The selection criteria were as follows:

1. The start of a storm was determined when the difference between two successive $D s t$ values (i.e. two successive hours) was greater than $15 \mathrm{nT}$. (Here, and in the following text, a change in Dst will be stated as a positive number although it is understood that an increase in $D s t$ would be seen as a more negative value.)

2. When averaging the first three $D s t$ values to form a quantity to be correlated with $K p$, it was required that all three values form a sequence of increasing Dst. Thus, if the start of the storm was at 0400 UT, the values of $D s t$ for the intervals 0400-0500 UT and 0500-0600 UT had to be discarded as the first hour of the $K p$ interval was not part of the storm main phase.

3. No positive $D s t$ values were used in computing a three hour average which was to be correlated with $K p$.

4. A fluctuation with a magnitude $<10 \mathrm{nT}$ was accepted in a three hour average of Dst. That is, if within a storm main phase Dst had three sequential hourly values of $-35 \mathrm{nT},-27 \mathrm{nT}$ and $-45 \mathrm{nT}$ these three values would have been averaged to form an acceptable data point for the study. If, on the other hand, the second value in the sequence had been $-24 \mathrm{nT}$ instead of $-27 \mathrm{nT}$, the three hour average value would not have formed part of our data base.

5 . The end of a storm recovery phase was defined as the time when three consecutive values of $D s t$ deviated from one another by $<5 \mathrm{nT}$.

6. $\delta(D s t)$ was the difference between the first and third values of $D s t$ in the three hour interval covered by $K p$.

I shall present the results in graphical form showing two types of plots. The first type shows all data points with different symbols used for the intervals 1987-1989, 1990-1992 and 1993-1996 inclusive. This permits the reader to see any differences in behaviour which might be apparent during the rise towards sunspot maximum (1987-1989), the period of solar maximum (1990-1992) and the period of decay towards sunspot minimum 
(1993-1996). The second type of display shows the average values of the data displayed in the aforementioned plots, which each data point comprising the average of equal numbers of original Dst data points. I shall show plots that pertain to the main phase growth and the recovery phase, and I shall break the latter into periods early and late in that phase. Finally, I shall present plots which relate both $D s t$ and $\delta(D s t)$ to the level of substorm EP activity as characterized by $K p$.

\section{Presentation of the data}

In this section I shall present all the comparisons between $K p$ and $D s t$, commenting briefly on the key elements of interest in each plot. I shall then summarize the findings linking them in the Discussion section that follows. In all the plots, the use of the term "increase" as applied to Dst means that the index is becoming more negative.

Figure 1a shows all the data points from 1987-1996 which describe the relationship between $K p$ and $D s t$ for main phase conditions while Fig. $1 \mathrm{~b}$ shows averages of the data so that each point represents equal numbers of $D s t$ values. Clearly $D s t$ increases with increasing $K p$ and it is also apparent that during the storm main phase there are no $K p$ values less than $4+$. Figure 2 describes the same data set but shows the relationship between the increase in $D s t[\delta(D s t)]$ and $K p$. Here one can see only a marginal tendency for $D s t$ to exhibit and increase with an increasing level of substorm activity as quantified by $K p$.

Figure 3a shows all the data points from 1987-1996 which describe the relationship between $K p$ and $D s t$ for recovery phase conditions while Fig. 3 b shows averages of the data so that each point represents equal numbers of $D s t$ values. While there are now many more low $K p$
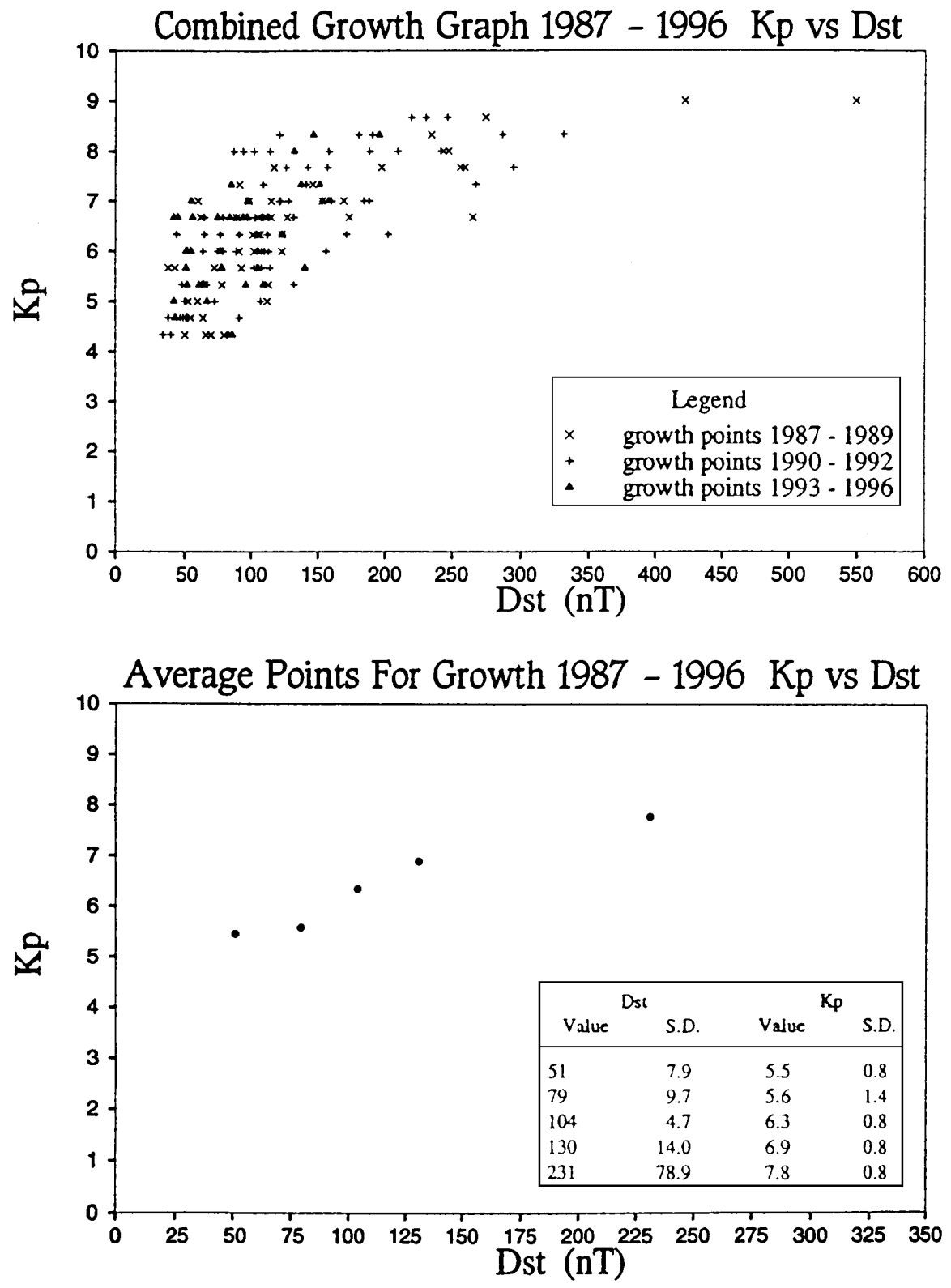

Fig. 1. a Shows Dst plotted as a function of $K p$ for storm main phase conditions over the period 1987-1996 inclusive. The data points for the period of rise toward sunspot maximum (1987-1989), the interval around sunspot maximum (1990-1992) and the period of decline towards sunspot minimum (1993-1996) are shown as different symbols. b Shows the data averaged for Dst bins of equal numbers of data points. Values for the data points and their standard deviations are shown in the right corner of the panel. There is a general tendency for $D s t$ to increase with increasing $K p$. Note that there are no cases where $K p<40$ for storm main phase conditions 

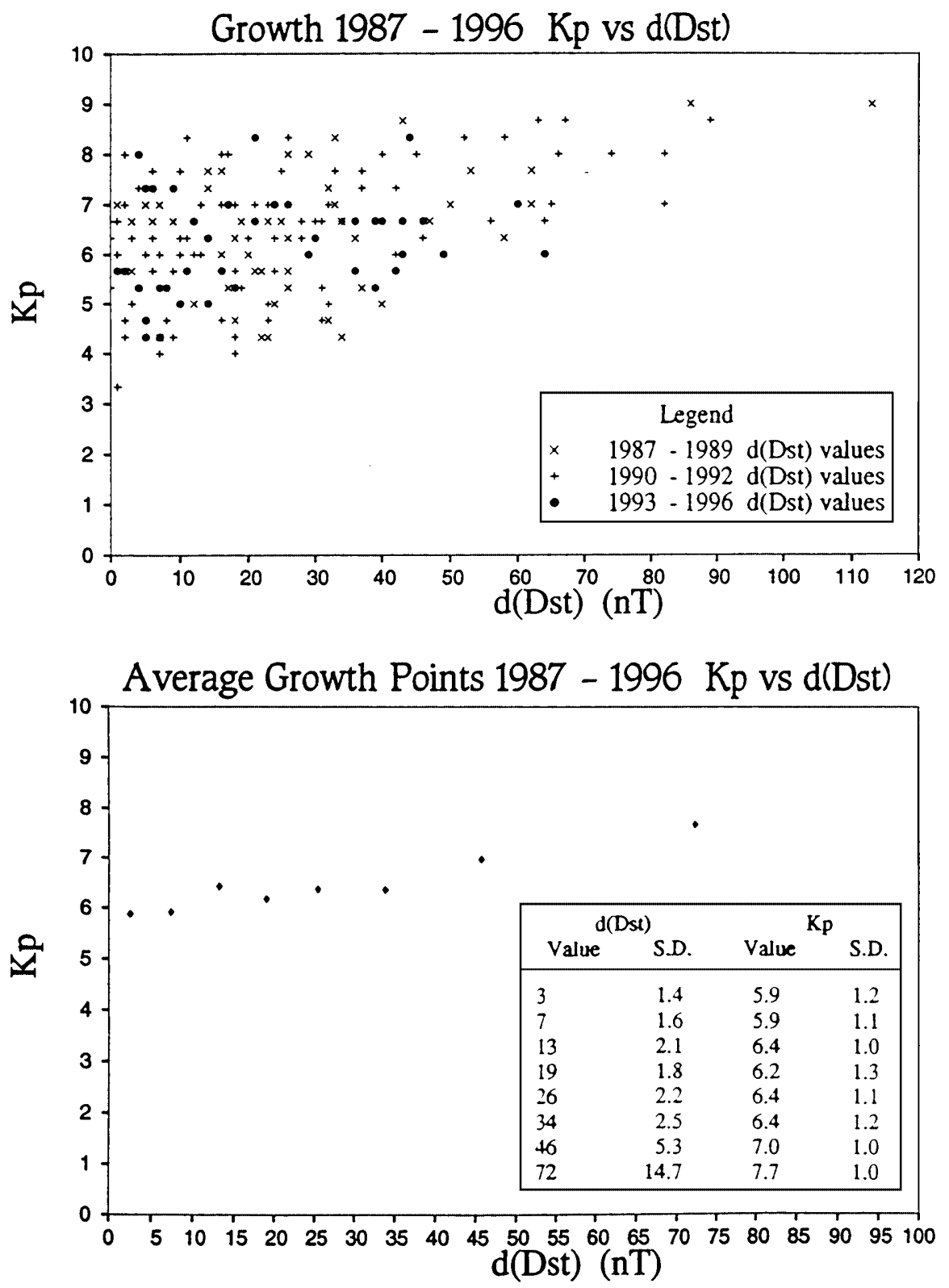

Fig. 2a, b. Same as Fig. 1 except for rate of increase of $D s t$ as a function of $K p$ for storm main phase conditions. There is very little relationship visible between the strength of substorm activity and the rate of increase of Dst points compared to the situation for main phase conditions, there is a clear and surprising tendency for $D s t$ to be higher for higher $K p$ values. Figure 4 describes the same data set but shows the relationship between the increase in $D s t[\delta(D s t)]$ and $K p$. Here there is much less of a trend, although large declines in Dst appear to be associated with higher $K p$.

This rather interesting result for recovery phase conditions prompted me to look for any difference in the relationship between $D s t$ and $K p$ for early recovery phase conditions compared to conditions later in the recovery phase. For this purpose, early recovery phase was defined as the first three hour period after the peak of the main phase and would include the second three hour period if Dst had decayed by less than $10 \mathrm{nT}$.

Figure 5a shows all the data points from 1987-1996 which describe the relationship between $K p$ and $D s t$ for early recovery phase conditions while Fig. 5b shows averages of the data so that each point represents equal numbers of $D s t$ values. These plots can be compared to the corresponding plots in Fig. 6 which describe the relationship between $K p$ and $D s t$ for late recovery phase conditions. It does not seem to matter whether one is experiencing early or late recovery phase conditions in that the relationship between $K p$ and $D s t$ seems very similar for both cases.

\section{Discussion of the results}

This research was started with the aim of trying to see if there was any clear relationship between substorm EP activity (as quantified by the $K p$ index) and the development of the symmetric ring current (as quantified by the Dst index). To achieve any success in such an effort is not a simple matter, since it is quite possible to 

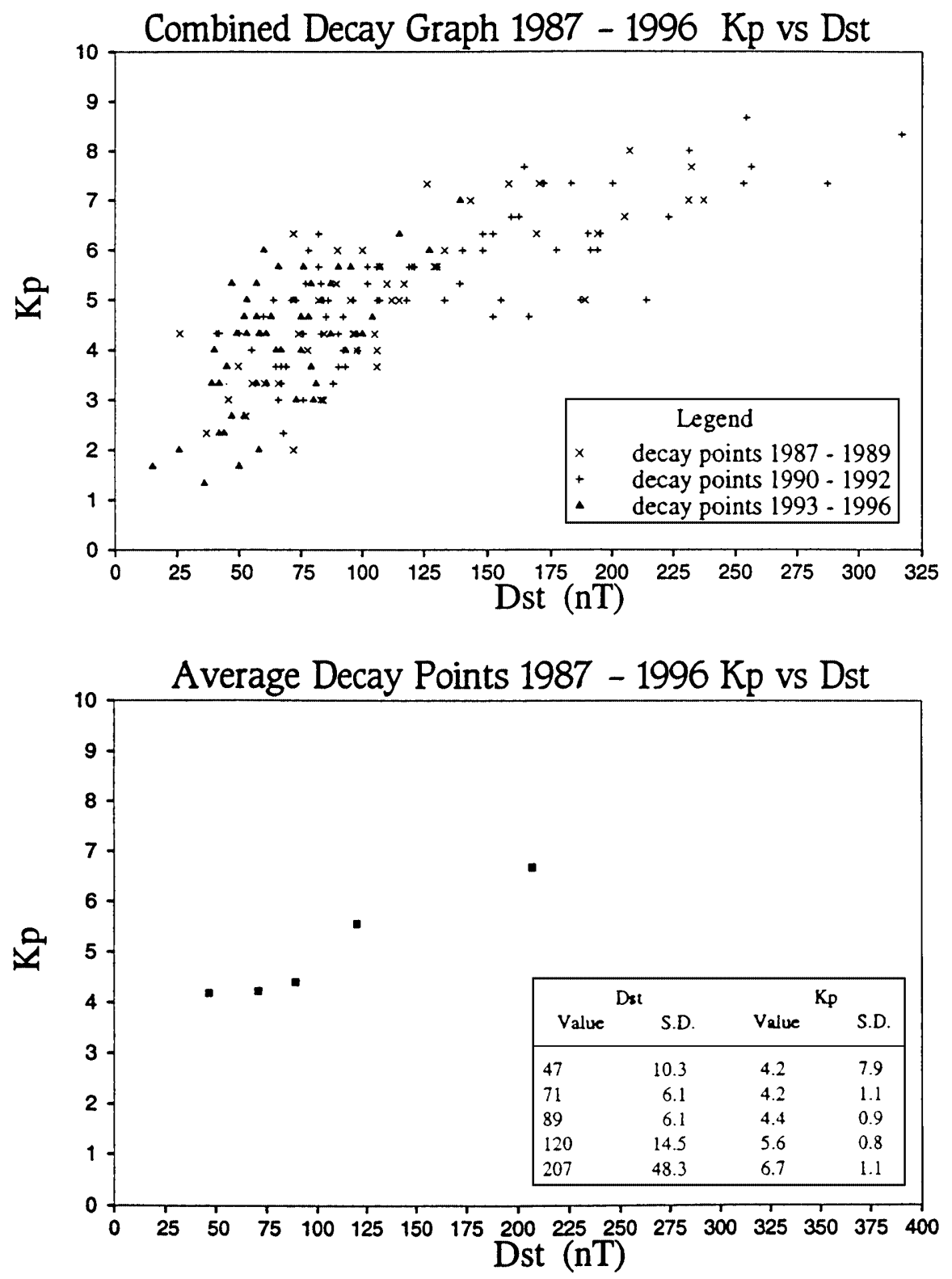

Fig. 3a, b. Same as Fig. 1 except for storm recovery phase conditions. There is a clear tendency for Dst to increase with increasing $K p$, which is not what one might have expected for recovery phase conditions imagine a situation in which changes in Dst and $K p$ seem to be correlated and yet an increase in symmetric ring current may not be attributable to the effects of substorm EP activity. This could arise if substorms and the symmetric ring current derive their energy from the same source (i.e. solar wind plasma and field) but were unrelated causally to one another. If that were the case, an increase in Dst would be expected to be accompanied by an increase in $K p$. A further complication is that magnetic perturbations associated with a substorm EP can contaminate the Dst index by contributing positive H-component disturbances from the current wedges that cannot easily be removed from the index given its present method of calculation and the number and distribution of contributing stations (Friedrich et al., 1999).

With these constraints in mind, the fact that Dst increases with increasing $K p$ during the main phase (Fig. 1) tells us nothing of significance regarding any possible causal relationship between substorm EP activity and ring current growth. Furthermore, the fact that $\delta(D s t)$ increases marginally with increasing $K p$ is equally uninformative insofar as any causal relationship between substorm EP activity and symmetric ring current growth is concerned. It is interesting to note that, for the Dst index to show any significant growth, $K p$ must be quite large. During main phase conditions, it can be seen from Fig. 1a that $K p>4^{\circ}$ at all times. This indicates that either substorm expansive phases during the main phase involve very large magnetic perturbations or the auroral electrojets and their associated field-aligned currents move to low latitudes so they can affect the $K p$ stations (or both these situations may occur). It is also clear that an average $K p$ of $6^{\circ}-7^{\circ}$ may characterize a wide range of $D s t$ increases over a three hour period ranging from a few $\mathrm{nT}$ to a few tens of $\mathrm{nT}$. This latter observation suggests that increases in Dst are unrelated to the strength of the accompanying substorm EP activity. 

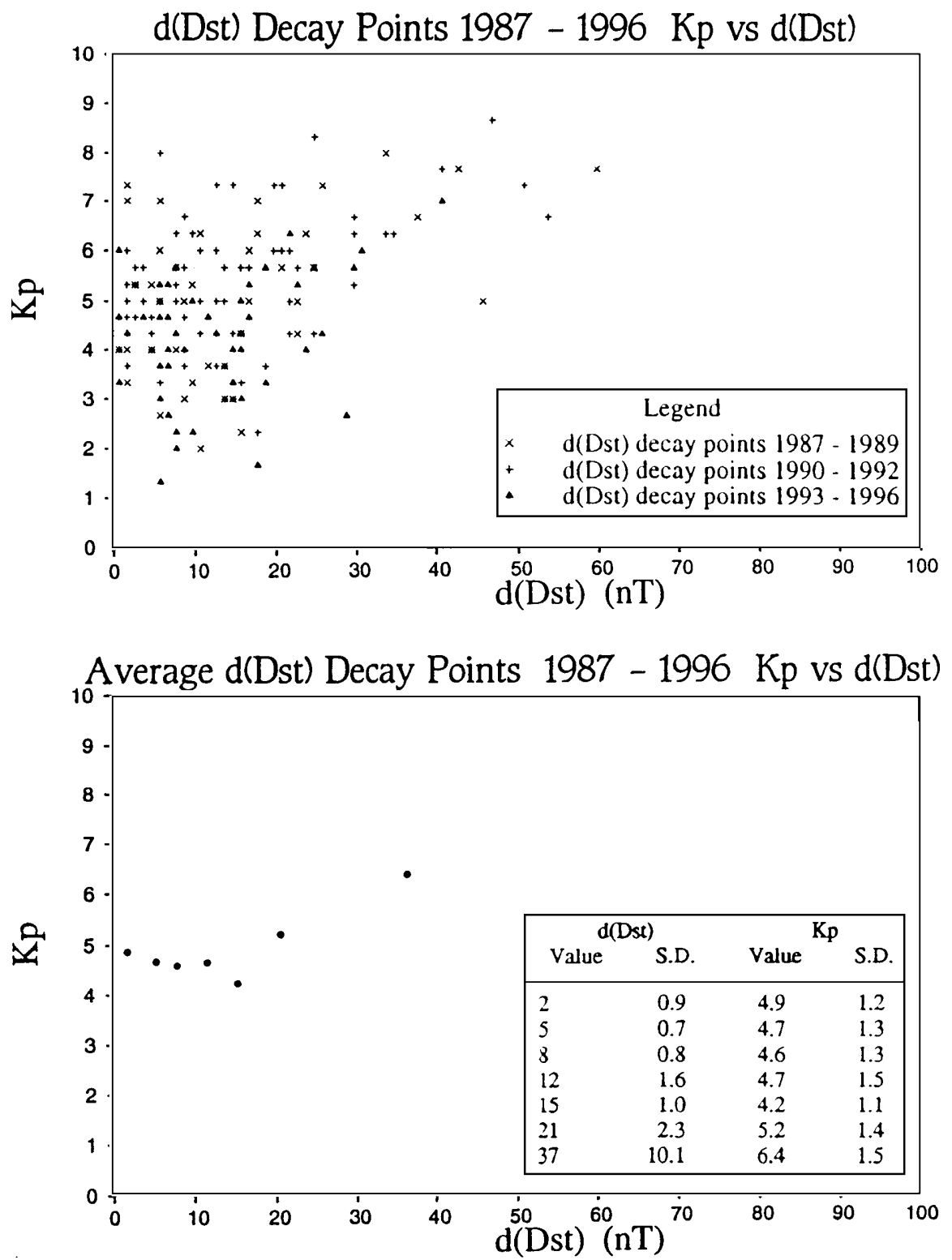

Fig. 4a, b. Same as Fig. 2 except for storm recovery phase conditions. There seems to be very little relationship between the strength of $K p$ and the rate of decrease of Dst
As alluded to earlier, the most interesting result of this study is that the size of Dst seems to be related to the strength of the accompanying substorm EP activity during the storm recovery phase. This effect characterizes both the early and later stages of the recovery phase. One would have expected that, during the recovery phase, there would be no particular relationship between the level of substorm EP activity and the size of Dst, with the symmetric ring current dominating Dst and simply going through its decay process. One can think of two possible reasons for this recovery phase relationship between $K p$ and $D s t$.

1. Although the storm may be in the recovery phase, there may still be significant substorm EP activity in progress. If EP activity is, indeed, responsible for symmetric ring current growth, then continual EP activity may succeed in keeping $D s t$ higher than it would have been if substorm activity had dropped to low levels immediately after main phase growth had ceased.
2. There is actually a significant contribution to Dst from the crosstail current as suggested by Alexeev et al. (1996) and this crosstail current is high during times of high substorm activity even in a storm recovery phase. Then the crosstail current makes Dst larger than would be expected assuming only the symmetric ring current to be responsible for that index. This, in turn, would lead one to believe that a significant portion of Dst may come from the contribution of the crosstail current even during the main phase of magnetic storms.

Either of these interpretations is consistent with the relationships between $K p$ and $D s t$ for the early and later phases of recovery as seen in Figs. $5 \mathrm{~b}$ and $6 \mathrm{~b}$. Of the two possible explanations, I believe the second one to be more likely. This is because one can sometimes observe a significant increase in Dst for cases of strong activity in which the EP disturbances are rather muted in the presence of strong directly driven activity (i.e. during 

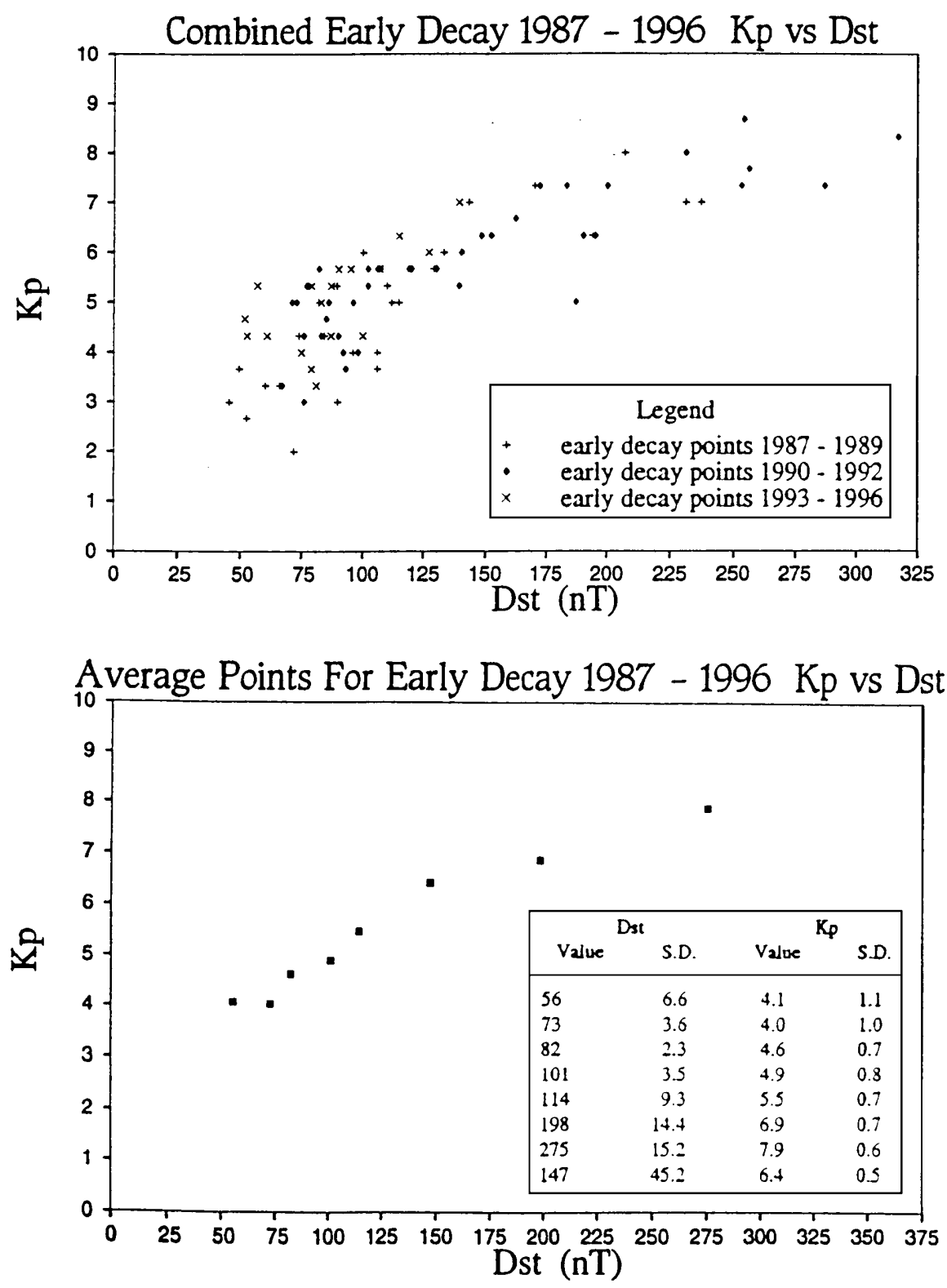

Fig. 5a, b. Same as Fig. 3 except for early recovery phase conditions. The tendency for high $D s t$ to be associated with high $K p$ as seen in Fig. 3 is confirmed convection bays). Figure 7 presents just such an example, in which a well-defined interval of strong southward IMF between $\sim 0700-1200$ UT results in a convection bay with only low level pseudo-breakup types of substorm EP activity being evident. The IMF $B z$ component fluctuates between northward and southward after 1200 UT and the convection bay declines in strength accompanied by more significant EP activity (with a particularly strong EP occurring shortly after 1200 UT). From Fig. 7, it can be seen that Dst tracks $K p$ very well. In this case, if Dst were produced by a symmetric ring current, one would expect it to decay on a much longer time scale than $K p$. This suggests that enhanced crosstail current produced by the increased energy flow into the magnetosphere due to the steady strong southward IMF produces a sufficiently large H-component perturbation at the Earth's surface to account for most of the Dst disturbance. The magnetic field data from the GOES 9 geostationary satellite (which is in the evening and midnight sector during most of this event) confirms this view. The third panel in Fig. 7 shows the deviation of the measured GOES $9 \mathrm{Bz}$ magnetic field from the quiet time value every hour on the hour. Clearly, this disturbance tracks Dst very nicely with a magnitude not far different from Dst itself. (Note that Dst is not corrected for Earth induction, so one would expect the source field to be weaker than the calculated Dst). Based on these data, it is reasonable to assume that most of the observed Dst response is due to the rise and fall of the crosstail current and little can be attributed to a true symmetric ring current which would be expected to have a much longer decay time dependent on charge exchange processes.

In concluding this section, I should like to point out the apparent trend for the correlation between $K p$ and $D s t$ to be more evident for recovery phase periods than 

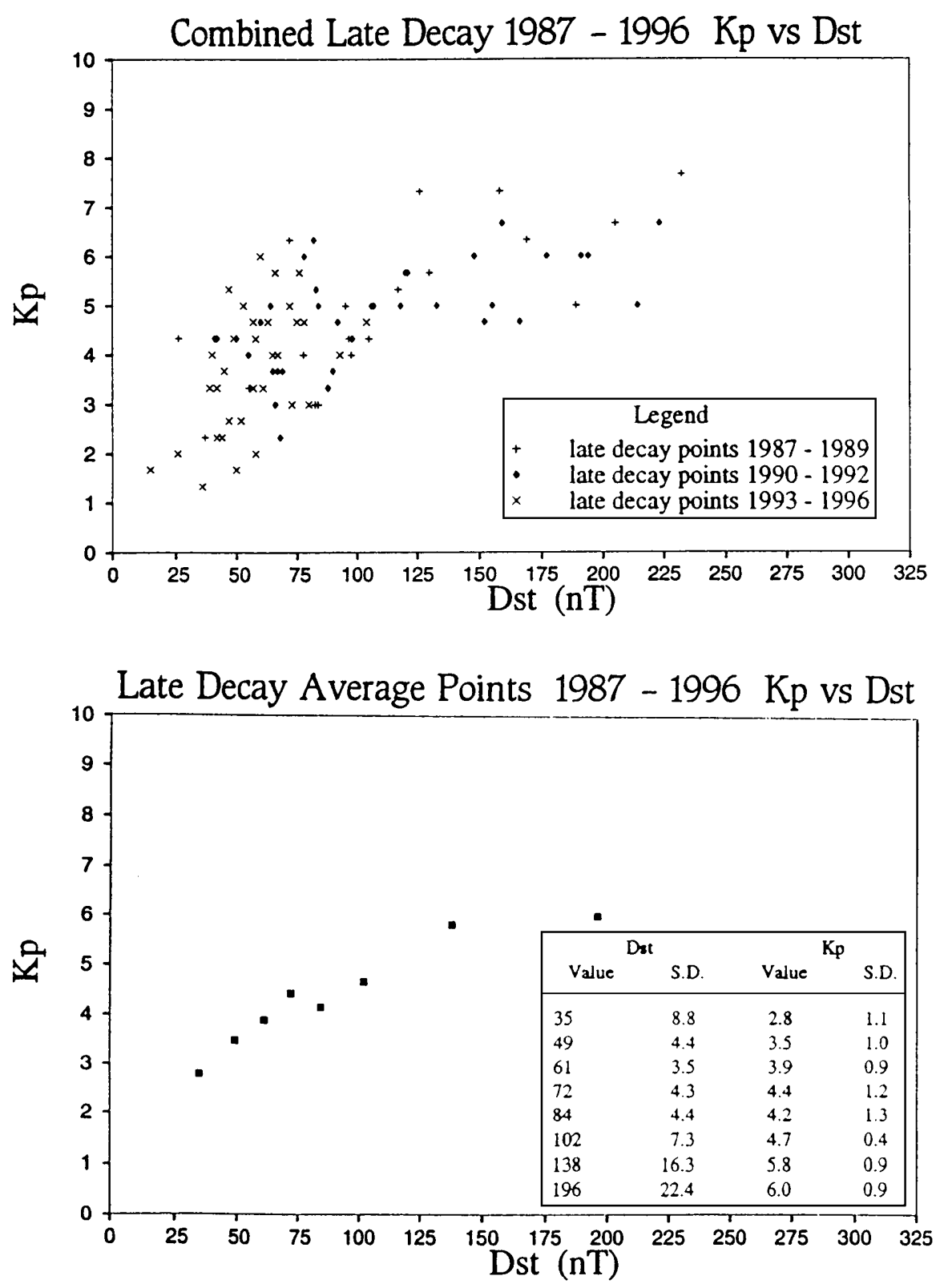

Fig. 6a, b. Same as Fig. 3 except for late recovery phase conditions. The tendency for high $D s t$ to be associated with high $K p$ in Fig. 3 is again confirmed, although the values of $K p$ for a given $D s t$ seem to be smaller than for early recovery phase conditions for main phase growth periods. This should not be considered as a significant point, as the trend for main phase growth periods is confined to a narrow range of $K p$ values in excess of $4^{\circ}$. In contrast, recovery phase periods have many low $K p$ values and this has the effect of accentuating the correlation. It would be useful, in the future to inspect the correlation between ap (the linear counterpart of $K p$ ) and $D s t$ to see if it is more compelling to the eye for both growth and recovery phase periods.

\section{Conclusions}

While these results contribute little to answering the question of whether or not substorm EP activity is responsible for the growth of the storm main phase symmetric ring current, it has produced some important clues about how changes in Dst should be interpreted. The clear trend for higher Dst to be associated with higher $K p$ during the storm recovery phase, regardless of whether it is early or late in the process, strongly suggests that a significant portion of the Dst disturbance may be due to contributions of the crosstail current. This is consistent with the earlier view expressed by Campbell (1973) that tail current effects might be more important than ring current effects for stations on the nightside. The effect may be even larger during the main phase when strong intensifications of the crosstail current near the inner edge of the current sheet (Kaufmann, 1987) may lead to transient increases in Dst of tens of nT during the growth phase of substorms.

If $D s t$ is going to continue to be used as an index to characterize the strength of the symmetric ring current, 


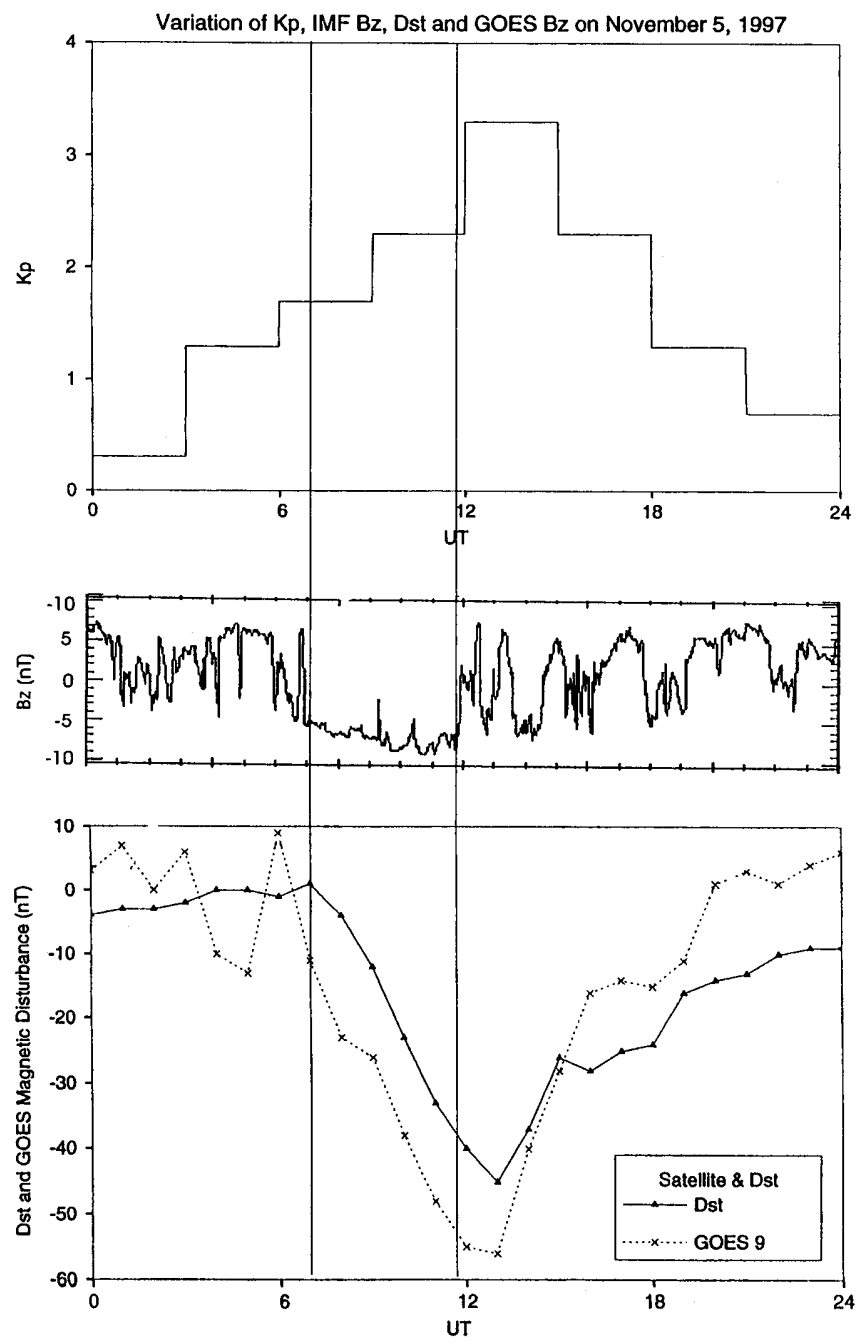

Fig. 7. Variation of $D s t$ and the GOES $9 \mathrm{Bz}$ component (bottom panel), the $B z$ component of the IMF detected by the WIND satellite (middle panel) and the Kp index (top panel) on November 5, 1997. Kp, $D s t$ and the $B z$ component of GOES 9 track one another rather well, suggesting that Dst is caused by crosstail current changes rather than by the growth and decay of a symmetric ring current

it seems all the more important now to quantitatively assess the various ways in which the index can be contaminated by contributions due to different sources. Campbell (1996) has recently argued that Dst is a quite imperfect index as a measure of the strength of the symmetric ring current, with many different factors contributing to the imperfections. This includes the effect of the substorm current wedge (Friedrich et al., 1999), the effect of the near-Earth crosstail current (Campbell, 1973; Alexeev et al., 1996) and the effect of the asymmetric ring current associated with directly driven activity (Kamide and Fukushima, 1972). Added to this is the strong possibility that there might be overhead ionospheric currents from systems such as DP 2 or systems encompassing the equatorial electrojet that contribute significant perturbations to mid- and lowlatitude stations on the day side. Until one is able to decouple the various competing contributions to Dst from the various sources of $\mathrm{H}$ - component magnetic disturbance at low latitudes, it will be impossible to confidently define the effect of substorm EP activity on the growth of the stormtime ring current.

Acknowledgements. I am grateful to Michelle Vigeant for carrying out the data analysis and preparation of the plots displaying in this paper. Ms. Vigeant was supported by the WISEST (Women in Scholarship, Engineering, Science and Technology) program at the University of Alberta. The permission of $\mathrm{H}$. Singer for use of the GOES 9 data and to the WIND Science Team for use of the WIND data is gratefully acknowledged. This research was supported, in part, by the Natural Sciences and Engineering Research Council of Canada.

Topical Editor G. Chanteur thanks W. H. Campbell and L. Zhu for their help in evaluating this paper.

\section{References}

Akasofu, S.-I., and S. Chapman, The ring current, geomagnetic disturbance and the Van Allen radiation belts, J. Geophys. Res., 66, 1321, 1961.

Alexeev, I. I., E. S. Belenkaya, V. V. Kalegaev, Y. I. Feldstein, and A. Grafe, Magnetic storms and magnetotail currents, J. Geophys. Res., 101, 7737, 1996.

Burton, R. K., R. L. McPherron, and C. T. Russell, An empirical relationship between interplanetary conditions and Dst, J. Geophys. Res., 80, 4102, 1975.

Cade III, W. B., J. J. Sojka, and L. Zhu, A correlative comparison of the ring current and auroral electrojets using geomagnetic indices, J. Geophys. Res., 100, 97, 1995.

Campbell, W. H., The field levels near midnight at low and equatorial stations, J. Atmos. Terr. Phys., 35, 1127, 1973.

Campbell, W. H., Occurrence of $A E$ and Dst geomagnetic index levels and the selection of the quietest days in a year, J. Geophys. Res., 84, 875, 1979.

Campbell, W. H., Geomagnetic storms: the Dst-ring current myth and lognormal distributions, J. Atmos. Terr. Phys., 58, 1171, 1996.

Friedrich, E., G. Rostoker, M. G. Connors, and R. L. McPherron, Influence of the substorm current wedge on the Dst index, J. Geophys. Res., 104, 4567, 1999.

Iyemori, T., and D. R. K. Rao, Decay of the Dst field of geomagnetic disturbance after substorm onset and its implication to storm-substorm relation, Ann. Geophysicae, 14, 608, 1996.

Joselyn, J. J., and B. T. Tsurutani, Geomagnetic sudden impulses and sudden storm commencements, Eos, 71, 1808, 1990.

Kamide, Y., and N. Fukushima, Positive geomagnetic bays in evening high latitudes and their possible connection with the partial ring current, Rep. Ionos. Space Res., Japan, 26, 79, 1972.

Kaufmann, R. L., Substorm currents: Growth phase and onset, J. Geophys. Res., 92, 7471, 1987.

Lui, A. T. Y., R. W. McEntire, and S. M. Krimigis, Evolution of the ring current during two geomagnetic storms, J. Geophys. Res., 92, 7459, 1987.

Rostoker, G., E. Friedrich, and M. Dobbs, Physics of magnetic storms, in Magnetic storms, Eds. B. T. Tsurutani, W. D. Gonzalez, Y. Kamide and J. K. Arbello, Geophys. Monograph, 98, p. 149, American Geophysical Union, 1997.

Singer, S. F., A new model of magnetic storms and aurorae, Trans. Am. Geophys. Un., 38, 175, 1957.

Sugiura, M., Hourly values of equatorial Dst for IGY, in Ann. Int. Geophys. Year, 35, p. 945, Pergammon Press, Oxford, 1964. 